Published in final edited form as:

Heart Rhythm. 2014 June ; 11(6): 925-932. doi:10.1016/j.hrthm.2014.03.006.

\title{
Use and Outcomes of Antiarrhythmic Therapy in Patients with Atrial Fibrillation Receiving Oral Anticoagulation: Results from the ROCKET AF Trial
}

\author{
Benjamin A. Steinberg, MD*, Anne S. Hellkamp, MS ${ }^{\star}$, Yuliya Lokhnygina, PhD*, Jonathan L. \\ Halperin, MD ${ }^{\dagger}$, Günter Breithardt, MD $\ddagger$, Rod Passman, MD§, Graeme J. Hankey, MD\|, \\ Manesh R. Patel, MD*, Richard C. Becker, MDף, Daniel E. Singer, MD\#, Werner Hacke, MD, \\ PhD $^{\star \star}$, Scott D. Berkowitz, MD ${ }^{\dagger \dagger}$, Christopher C. Nessel, MD ${ }^{\ddagger}$, Kenneth W. Mahaffey, MD§§, \\ Keith A.A. Fox, MB ChB $\|^{\| l}$, Robert M. Califf, MDII, and Jonathan P. Piccini, MD ${ }^{\star}$ on behalf of \\ the ROCKET AF Steering Committee and Investigators \\ *Duke Clinical Research Institute, Duke University Medical Center, Durham, NC \\ †Cardiovascular Institute, Mount Sinai Medical Center, New York, NY

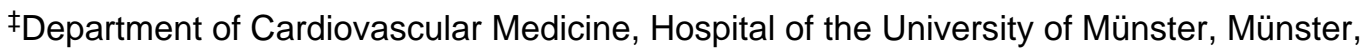 \\ Germany \\ §Northwestern University Feinberg School of Medicine \\ "School of Medicine and Pharmacology, The University of Western Australia, Australia \\ IUniversity of Cincinnati College of Medicine, Cincinnati, $\mathrm{OH}$
}

(C) 2014 The Heart Rhythm Society. Published by Elsevier Inc. All rights reserved.

Corresponding author: Benjamin A. Steinberg, MD, Duke Clinical Research Institute, PO Box 17969, Durham, NC 27715. Phone: (919) 668-8686; Fax: (877) 991-8498. benjamin.steinberg@dm.duke.edu.

Conflict of interest: Steinberg: Dr. Steinberg was funded by NIH T-32 training grant \#5 T32 HL 7101-38. However, no relationships exist related to the analysis presented. Hellkamp: Nothing to disclose. Lokhnygina: Nothing to disclose. Halperin: Honoraria and served as a consultant and on an advisory board for Bayer and Johnson \& Johnson. Breithardt: Honoraria from Johnson \& Johnson and Bayer; and advisory board fees from Boehringer Ingelheim, Bristol-Myers Squibb, Pfizer, and Sanofi-Aventis. Passman: Speaker's bureau fees from Boehringer Ingelheim, Johnson and Johnson, and Medtronic Inc. and research support from Medtronic Inc. Dr. Passman is funded by the NIH R34 HL113404-01. Hankey: Honoraria for serving on executive committees for Johnson \& Johnson and Bayer. Patel: Honoraria from Johnson \& Johnson and Bayer HealthCare for serving on the executive committee of the ROCKET AF trial; consulting fees from Ortho McNeil Janssen, and Bayer HealthCare; and advisory board fees from Genzyme. Becker: Research support from Bayer and Johnson \& Johnson. Singer: Consultant and/or member of a scientific advisory board for Bayer, Boehringer Ingelheim, Bristol-Myers Squibb, Daiichi Sankyo, Johnson \& Johnson, and Pfizer; has an institutional research support contract with Johnson \& Johnson; served as an unpaid ROCKET AF executive committee member. Hacke: Honoraria for serving on an executive committee for Johnson \& Johnson and Bayer. Berkowitz: Full-time employee of Bayer HealthCare Pharmaceuticals. Nessel: Full-time employee of Janssen Research \& Development LLC. Mahaffey: Grant support (significant) from AstraZeneca, Bayer, Boehringer Ingelheim, Bristol-Myers Squibb, Daiichi Sankyo, Eli Lilly, GlaxoSmithKline, Johnson \& Johnson, Merck, Momenta Pharmaceuticals, Novartis, Portola, Pozen, Regado Biotechnologies, Sanofi-Aventis, Schering-Plough (now Merck), The Medicines Company; consulting fees (significant) from AstraZeneca and Johnson \& Johnson, (modest) Bayer, Boehringer Ingelheim, Bristol-Myers Squibb, Daiichi Sankyo, Eli Lilly, GlaxoSmithKline, Merck, Novartis, Ortho/McNeill, Pfizer, Polymedix, Sanofi-Aventis, Schering-Plough (now Merck). Fox: Grant funding and honoraria from Bayer and Johnson\& Johnson. Califf: Consulting fees and research funding from Johnson \& Johnson; all other industry interactions are listed at www.dcri.org/about-us/ conflict-of-interest. Piccini: Grants for clinical research from ARCA Biopharma, Boston Scientific, GE Healthcare, Johnson \& Johnson, and Resmed. Consulting to BMS/Pfizer, Johnson \& Johnson, Forest Laboratories, Medtronic, and Spectranetics.

Publisher's Disclaimer: This is a PDF file of an unedited manuscript that has been accepted for publication. As a service to our customers we are providing this early version of the manuscript. The manuscript will undergo copyediting, typesetting, and review of the resulting proof before it is published in its final citable form. Please note that during the production process errors may be discovered which could affect the content, and all legal disclaimers that apply to the journal pertain. 
\#Massachusetts General Hospital and Harvard Medical School, Boston, MA

${ }^{* *}$ Ruprecht-Karls-University, Heidelberg, Germany

${ }^{\dagger+}$ Bayer HealthCare Pharmaceuticals, Montville, NJ

抽anssen Research \& Development LLC, Raritan, NJ

§Department of Medicine, Stanford University, Stanford, CA

IIIInniversity of Edinburgh and Royal Infirmary of Edinburgh, Edinburgh, UK

IIIDuke Translational Medicine Institute, Duke University Medical Center, Durham, NC

\section{Abstract}

Background-Antiarrhythmic drugs (AAD) and anticoagulation are mainstays of atrial fibrillation (AF) treatment.

Objective-We aimed to study the use and outcomes of AAD therapy in anticoagulated AF patients.

Methods-Patients in the ROCKET AF trial $(\mathrm{n}=14,264)$ were grouped by AAD use at baseline: amiodarone, other AAD, or no AAD. Multivariable adjustment was performed to compare stroke, bleeding, and death across groups, as well as across treatment assignment (rivaroxaban or warfarin).

Results-Of 14,264 patients randomized, 1681 (11.8\%) were treated with an AAD (1144 [8\%] with amiodarone, 537 [3.8\%] with other AADs). Amiodarone-treated patients were less-often female ( $38 \%$ vs. $48 \%$ ), had more persistent AF ( $64 \%$ vs. $40 \%$ ), and more concomitant heart failure ( $71 \%$ vs. $41 \%$ ) than patients receiving other AADs. Patients receiving no AAD more closelyresembled amiodarone-treated patients. Time in therapeutic range was significantly lower in warfarin-treated patients receiving amiodarone versus no AAD (50\% vs. $58 \%$, p<0.0001). Compared with no AAD, neither amiodarone (adjusted HR 0.98, 95\% CI 0.74-1.31, $\mathrm{p}=0.9$ ) nor other AADs (adjusted HR 0.66, 95\% CI 0.37-1.17, p=0.15) were associated with increased mortality. Similar results were observed for embolic and bleeding outcomes. Rivaroxaban treatment effects in patients not on an AAD were consistent with the overall trial (primary endpoint adjusted HR $0.82,95 \%$ CI $0.68-0.98$, pinteraction $=0.06$; safety endpoint adjusted HR 1.12 , $95 \%$ CI $0.90-1.24$, pinteraction $=0.33$ ).

Conclusion-Treatment with AADs was not associated with increased morbidity or mortality in anticoagulated patients with AF. The influence of amiodarone on outcomes in patients receiving rivaroxaban requires further study.

\section{Keywords}

atrial fibrillation; antiarrhythmic drugs; rivaroxaban; warfarin; outcomes

\section{INTRODUCTION}

The treatment of patients with atrial fibrillation (AF) focuses on 3 primary objectives: (1) prevention of stroke and systemic embolism, (2) control of ventricular rate, and (3) 
treatment of symptoms. Medical therapy remains a mainstay for each of these goals, and frequently requires antiarrhythmic drug (AAD) therapy and oral anticoagulation. However, these drug groups present specific management challenges, as well as interactions that may mitigate effectiveness and/or increase the risk of adverse events. This is of particular interest for recently approved novel oral anticoagulants, which may lack many of the interactions that limit vitamin $\mathrm{K}$ antagonist (VKA) therapy.

Rivaroxaban is a novel, oral factor Xa inhibitor that is approved for the prevention of stroke or non-central nervous system (CNS) embolism in patients with nonvalvular AF. Its safety and efficacy were demonstrated in the ROCKET AF (Rivaroxaban Once Daily Oral Direct Factor Xa Inhibition Compared with Vitamin K Antagonism for Prevention of Stroke and Embolism Trial in Atrial Fibrillation) trial ${ }^{1}$. However, few data exist regarding the use of rivaroxaban in patients also receiving AAD therapy. The objectives of the current analysis were to: (1) assess clinical outcomes in patients treated with AAD therapy and concomitant anticoagulation, and (2) determine whether the treatment effect of rivaroxaban compared with warfarin varies with AAD therapy.

\section{METHODS}

The design of the ROCKET AF study has been described in detail previously (NCT00403767) ${ }^{2}$. Briefly, the ROCKET AF trial was a prospective, randomized, doubleblind, placebo-controlled trial of fixed-dose rivaroxaban versus adjusted-dose warfarin for the prevention of stroke or non-CNS systemic embolism in patients with nonvalvular AF at high risk of stroke. Patients underwent clinical assessment at a minimum of every 4 weeks throughout the trial, and this included medication reconciliation and ascertainment of interval events. The use of AAD therapy was at the discretion of the treating physician, and not blinded or randomized.

The present study is a post-hoc analysis including all patients randomized in the trial (intention-to-treat [ITT]), and subsequently grouped according to baseline use of a membrane-active AAD that is used clinically in the treatment of AF. These AADs included amiodarone, dronedarone, sotalol, dofetilide, propafenone, flecainide, quinidine, and disopyramide. After preliminary analyses revealed the majority of AAD use to be amiodarone, the population was stratified by amiodarone use, all other AAD use, and no $\mathrm{AAD}$ at baseline. Baseline characteristics and outcomes were compared among these groups. For patients on amiodarone, dosing distribution is presented using most recent reported dose.

Patients were included in the analysis as long as they remained in their baseline group. Patients who either discontinued AAD therapy or changed groups (from amiodarone to other $\mathrm{AAD}$, from other $\mathrm{AAD}$ to amiodarone, or from no AAD to any $\mathrm{AAD}$ ) were censored at the time of therapy change. For patients not on AAD at baseline, exposures of $<7$ days were ignored. For patients on any AAD at baseline, temporary interruptions of $<30$ days were ignored. For patients assigned to warfarin, time in therapeutic range (TTR) was calculated for the period of follow-up, during which the patient remained in the same group as baseline (amiodarone, other AAD, no AAD). 


\section{OUTCOMES}

Clinical endpoints in the ROCKET AF trial have been described previously ${ }^{2}$. The primary endpoint was the occurrence of stroke (ischemic or hemorrhagic) or non-CNS embolism and the primary safety endpoint was the composite of non-major clinically relevant (NMCR) and major bleeding as defined by the International Society on Thrombosis and Haemostasis (ISTH). The present analysis compared outcomes among AAD groups and according to treatment assignment (rivaroxaban or warfarin). Specifically, secondary outcomes of efficacy included composite and individual endpoints of stroke, non-CNS embolism, myocardial infarction (MI), or vascular death, as well as the individual endpoints of allcause death, non-vascular death, cardiac failure, hospitalization, and emergency department (ED) visits. As in prior analyses, 93 patients were excluded from the efficacy analysis due to violations of Good Clinical Practice at the enrolling center. Safety outcomes were also assessed, and limited to the on-treatment population (patients in the ITT population who received at least 1 study medication dose). These included major bleeding and/or NMCR bleeding.

\section{Statistical Methods}

Summary statistics are presented for patterns of AAD use, including proportions of patients, specific drug types, and exposure times. For patients on non-amiodarone AADs, patients who took more than 1 drug were counted for the type taken for the largest proportion of time.

Baseline characteristics are presented as percent (count) for categorical variables and as medians (25th, 75th percentiles) for continuous variables. Because these statistics are intended to describe the analysis population rather than to test any formal hypotheses, no pvalues are presented.

For amiodarone dosing, if more than 1 dose was indicated, the last dose was used. Amiodarone dose is reported in categories, but was tested as a continuous variable using Wilcoxon rank sum.

Median (25th, 75th percentile) TTR for each AAD group was calculated, and pairwise comparisons made using Wilcoxon rank sum tests. Only international normalized ratio (INR) values from the time period during which the patient was in the AAD group were used.

For all of the endpoints, event rates (events per 100 patient-years and total events) were generated. Groups were compared using Cox proportional hazards models. Efficacy endpoint models contained the following covariates: age, sex, body mass index, region, diabetes, prior stroke/transient ischemic attack (TIA), vascular disease (MI, peripheral arterial disease, carotid occlusive disease), congestive heart failure, hypertension, chronic obstructive pulmonary disease (COPD), paroxysmal AF, diastolic blood pressure (BP), creatinine clearance (Cockroft-Gault), ${ }^{3}$ heart rate, and abstinence from alcohol use. Safety endpoint models contained the following covariates: age; sex; region; prior stroke/TIA; anemia; prior gastrointestinal (GI) bleed; COPD; diastolic BP; creatinine clearance 
(Cockroft-Gault) ${ }^{3}$; platelets; albumin; and prior aspirin, VKA, or thienopyridine use.

Covariates were imputed where missing using the median for continuous variables and the mode for categorical variables, within groups of patients on or not on an AAD at baseline. Models also contained randomized treatment. Hazard ratios (with 95\% confidence interval $[\mathrm{CI}])$ and p-values are presented.

Because either new start or cessation of AAD therapy can be influenced by patient characteristics or intervening events that can also be related to the outcomes, patients were weighted by the inverse probability of continuing in their therapy group (see Supplemental Material).

For the recurrent events of hospitalizations and ED visits, we used the method of Wei, Lin, and Weissfeld for multiple failure times, with a robust sandwich variance estimator ${ }^{4}$. These models incorporated the weighting described above. Due to the small number of patients with repeated events, these models included first and second hospitalizations and first and second ED visits only. A single weighted parameter estimate was used to generate a significance test (Z-score) as well as hazard ratio estimate and CI.

For the primary efficacy and safety endpoints, as well as the specific bleeding endpoints (major, intracranial, GI, fatal, and NMCR), event rates (events per 100 patient-years and total events) were also generated by treatment arm and amiodarone use (vs. no AAD). Patients in the other AAD group were not used in these calculations. Cox models were constructed as above, with the addition of an amiodarone-by-treatment interaction term. Rivaroxaban versus warfarin hazard ratios (95\% CIs) were generated for the AAD groups, and the interaction p-value is reported.

All statistical analyses were performed by the Duke Clinical Research Institute (DCRI) using SAS software (version 9.2, SAS Institute, Cary, NC).

\section{RESULTS}

\section{Antiarrhythmic Use}

Of 14,264 patients randomized in ROCKET AF, 1681 (11.8\%) were on an AAD at baseline: $1144(8.0 \%)$ on amiodarone and $537(3.8 \%)$ on other AADs. Among patients on other AADs, 278 (52\%) received sotalol, 186 (35\%) propafenone, 58 (11\%) flecainide, 7 (1.3\%) quinidine, and $4(0.7 \%)$ each received disopyramide or dofetilide. No patient was on dronedarone. Derivation of the study population and persistence of therapies are shown in Figure 1. Similar treatment duration and AAD discontinuation rates were observed between patients on amiodarone (median 20 months, 21\% discontinuation) and other AADs (median 21 months, $22 \%$ discontinuation). Study drug discontinuation (rivaroxaban or warfarin) was similar in those treated with amiodarone versus no AAD (32\% for amiodarone, $34 \%$ for patients on no AAD, 26\% for other AADs).

\section{Patient Characteristics}

Characteristics of the patients, stratified by AAD group at baseline (amiodarone, other AAD, or no AAD), are shown in Table 1. Treatment assignment was balanced across AAD groups. 
Compared with patients not on AAD at baseline, those on other AADs were more often female ( $48 \%$ vs. $40 \%$ ), with higher rates of paroxysmal AF (59\% vs. $14 \%$ ), and lower rates of heart failure ( $41 \%$ vs. $63 \%$ ). Patients on amiodarone more closely resembled those not treated with an $\mathrm{AAD}$, however, they had higher rates of heart failure (71\% vs. 63\%), less prior VKA use (53\% vs. $63 \%$ ), and less digitalis use (24\% vs. $41 \%$ ) compared with patients not on an AAD.

Among amiodarone-treated patients, over 70\% in each group were treated with $200-300 \mathrm{mg}$ daily, followed by $100-150 \mathrm{mg}$ (14\% on rivaroxaban, $12 \%$ on warfarin), $400-500 \mathrm{mg}(9.1 \%$ rivaroxaban, $7.9 \%$ warfarin), and $600 \mathrm{mg}$ (2.3\% rivaroxaban, 3.3\% warfarin); doses of $<100$ $\mathrm{mg}$ or $>800 \mathrm{mg}$ were used in $1 \%$ or less, and there were no differences in dose between the rivaroxaban and warfarin groups $(\mathrm{p}=0.6)$. Complete TTR data are shown in Table 2. Among patients assigned to warfarin, the median TTR for patients receiving amiodarone (50\% [25th, 75th percentiles: 33, 64\%]) was significantly lower when compared with other AADs $(61 \%$ [45, 74\%], $\mathrm{p}<0.0001)$ and compared with patients on no AAD $(58 \%$ [43, 71\%], $\mathrm{p}<0.0001 ; \mathrm{p}=0.16$ for other AAD vs. no AAD). Extreme deviations in INR $(<1.5$ or $>4)$ were uncommon across all 3 groups ( $<5 \%$ of the time).

\section{Outcomes by Antiarrhythmic Group}

Adjusted efficacy and safety outcomes are shown in Table 3. Compared with patients on no AAD at baseline, those treated with amiodarone had an increased risk of incident MI (adjusted hazard ratio [HR] 1.76, 95\% CI 1.11-2.77, $\mathrm{p}=0.02$ ), however, they did not have a significantly different risk of any other efficacy or safety outcome. There was no evidence of increased mortality in those treated with amiodarone (HR 0.98, 95\% CI $0.74-1.31, \mathrm{p}=0.90$ ) (Figure 2). Similarly, patients treated with other AAD agents had a similar risk of major adverse events when compared with patients not on an AAD at baseline. Raw, unadjusted event rates are available in the Supplemental Material (Table S1).

\section{Outcomes by Treatment Assignment}

Kaplan-Meier curves for the primary endpoint in each of the 4 groups are shown in Figure 3. Adjusted outcomes comparing rivaroxaban- versus warfarin-assigned patients among patients on amiodarone and not on any AAD at baseline are shown in Table 4. Treatment effects of rivaroxaban versus warfarin in patients not on AAD therapy are consistent with results from the overall trial (stroke or non-CNS embolism adjusted HR 0.82, 95\% CI 0.680.98 , major bleeding adjusted HR $1.05,95 \%$ CI $0.90-1.24$ ). Among patients treated with amiodarone, there were low numbers of stroke or systemic embolic events (34 overall), and low numbers of major bleeding events ( 43 overall). This yielded wide confidence intervals around hazard estimates (adjusted HR for major bleeding 2.20, 95\% CI 0.98-4.91; adjusted HR for stroke or systemic embolism 1.71, 95\% CI 0.8-3.65). All tests of interaction between treatment assignment and AAD use were non-significant. In patients receiving amiodarone, there was no significant interaction between treatment assignment (rivaroxaban vs. warfarin) and renal dysfunction, for the primary endpoint $(\mathrm{p}=0.40)$. 


\section{DISCUSSION}

Of the 14,264 patients randomized in the ROCKET AF trial, a minority were treated with an $\mathrm{AAD}$ at baseline. However, amiodarone was the most common AAD used and patients treated with amiodarone were among the highest risk. These patterns are consistent with the indications and contraindications for AAD agents. Several of the non-amiodarone AADs require preserved renal function and are contraindicated in structural or ischemic cardiovascular disease. By comparison, amiodarone is often the only agent appropriate for medically-complex patients, or is reserved as the last option in patients with refractory AF due to toxicity profile. This is represented in our data, as the amiodarone group closely resembles those patients not on any AAD rather than those patients on an alternative AAD. Furthermore, the amiodarone group carried a striking proportion of heart failure patientsover $70 \%$ had heart failure versus $63 \%$ for no AAD and $41 \%$ for other AADs. The majority of amiodarone patients were on a dose consistent with clinical treatment of AF (100-300 mg daily), yet we cannot confirm the indication was not ventricular tachycardia. Despite its shortcomings, amiodarone remains the primary AAD for patients with heart failure and AF.

The present analysis represents the largest patient-level study of TTR in those receiving concomitant warfarin and amiodarone, and provides additional insight into the adverse effect of amiodarone on clinical outcomes ${ }^{5,6}$. These patients had higher rates of both suband supra-therapeutic INRs, and TTR is well-known to correlate closely with both bleeding and ischemic outcomes ${ }^{7}$. However, despite the increased overall risk of patients on amiodarone and the lower TTR in these patients, our data did not show increased risk of morbidity or mortality associated with either of the AAD groups (compared with no AAD).

These data may seem counter to results from the AFFIRM trial (Atrial Fibrillation Followup Investigation of Rhythm Management) and others, which suggest AADs (amiodarone in particular) increase the risk of morbidity and mortality, specifically non-cardiovascular mortality 8,9 . Yet, the AFFIRM investigators partially attributed the lower survival to the lower rates of anticoagulation therapy in AAD-treated patients in their cohort. This was not the case in ROCKET AF. All patients received stroke prevention therapy (e.g., anticoagulation), and this suggests that perhaps an element of risk associated with AAD therapy could be reduced by using anticoagulation. This is an important message for clinicians managing patients with AF on AAD therapy as these patients frequently exhibit paroxysmal arrhythmia and may not manifest clinical AF during a visit. There is little evidence for withholding anticoagulation in such patients ${ }^{10}$.

In patients receiving no $\mathrm{AAD}$ at baseline, hazard ratios of treatment with rivaroxaban versus warfarin were consistent with results from the overall trial. Rivaroxaban was noninferior for the prevention of stroke and demonstrated a significant reduction in fatal and/or intracranial bleeding, at the expense of increased risk of GI bleeding. However, in patients receiving amiodarone, the hazard ratios trend the other way, suggesting increased risk of ischemic and bleeding outcomes in patients assigned to rivaroxaban versus warfarin, and a borderline pvalue for the interaction term (0.06). Importantly, interpretation of these results is limited primarily by power. Event rates in these groups are relatively low and yield wide confidence intervals; definitive conclusions about the treatment effects cannot be made. This is 
particularly evident in the fact that risk of fatal bleeding and risk of intracranial hemorrhage — events frequently linked—-trend in opposite directions.

Additionally, absolute rates of events in patients receiving rivaroxaban are similar irrespective of amiodarone treatment; in contrast, rates of events in patients receiving concomitant warfarin and amiodarone are lower (particularly ischemic events) than in patients receiving warfarin and no AAD. This discrepancy accounts for the difference in hazard ratios between patients treated and not treated with amiodarone. However, in a detailed analysis of TTR in patients receiving warfarin, volatility of INRs in the amiodarone group did not appear to account for this effect.

There is a pharmacokinetic interaction between these drugs: amiodarone is well-known to inhibit both P-glycoprotein and cytochrome P450 3A4 (CYP3A4). The current U.S. Food and Drug Administration label for rivaroxaban states that patients with renal impairment taking P-glycoprotein and weak-to-moderate CYP3A4 inhibitors (like amiodarone) may have increases in exposure, which may increase bleeding risk. The ROCKET AF trial protocol did not specifically dose adjust for such interactions ${ }^{11}$. Cardiovascular drugs affected by the P-glycoprotein system are well described and can alter clinical outcomes. Furthermore, such an effect is not limited to rivaroxaban-all oral anticoagulants, including warfarin, exhibit such interactions to varying degrees ${ }^{12}$. Therefore, while there remains the potential for clinically significant interactions between amiodarone and rivaroxaban, further studies are necessary to precisely define such an effect.

Lastly, our data highlight the limited use of rhythm control therapies in patients with AF, who are at high risk of stroke. A minority of patients in the ROCKET AF trial had a prior catheter ablation for $\mathrm{AF}$, and only 79 underwent such a procedure during the trial. ${ }^{13}$ Our analysis shows that only a minority received AAD therapy; and while amiodarone can be highly effective, it is also the most toxic AAD. Similar rates were observed in the ARISTOTLE trial - approximately 1 in 10 patients was treated with amiodarone. ${ }^{14}$ While these data may not reflect trends in the broader, general AF population, rhythm control strategies in such high-risk patients warrant further investigation. ${ }^{15}$

\section{LIMITATIONS}

The current study represents a post-hoc, subgroup analysis of the ROCKET AF trial. As such, it should be interpreted as hypothesis-generating. Treatment with AAD was not randomized and thus there may exist residual and/or unmeasured confounding in comparisons among AAD groups. Furthermore, the number of patients receiving amiodarone, although substantial (>1100), was a fraction of the overall trial, and thus may lack the power to precisely measure the treatment effect, if any, of rivaroxaban compared with warfarin. Lastly, we cannot exclude the use of amiodarone for ventricular arrhythmias.

\section{CONCLUSIONS}

A minority of patients in ROCKET AF were treated with an AAD. However, AAD therapy was not associated with worse clinical outcomes in anticoagulated patients with AF. 
Addition of amiodarone to warfarin significantly reduces TTR, and the effect of amiodarone on rivaroxaban effectiveness requires additional investigation.

\section{Supplementary Material}

Refer to Web version on PubMed Central for supplementary material.

\section{Acknowledgments}

Funding: This work was supported by Janssen Research \& Development LLC, Raritan, NJ; and Bayer HealthCare AG, Leverkusen, Germany. Dr. Steinberg was funded by NIH T-32 training grant \#5 T32 HL 7101-38. Dr. Passman is funded by the NIH R34 HL113404-01.

\section{Abbreviations}
AAD
antiarrhythmic drug
AF
atrial fibrillation
BP
blood pressure
CI
confidence interval
CNS
central nervous system
COPD
chronic obstructive pulmonary disease
ED
emergency department
GI gastrointestinal
HR hazard ratio
INR international normalized ratio
ISTH International Society on Thrombosis and Haemostasis
ITT intention to treat
MI myocardial infarction
NMCR non-major clinically relevant
ROCKET AF Rivaroxaban Once Daily Oral Direct Factor Xa Inhibition Compared with Vitamin K Antagonism for Prevention of Stroke and Embolism Trial in Atrial Fibrillation
TIA transient ischemic attack
TTR time in therapeutic range
VKA vitamin $\mathrm{K}$ antagonist

\section{References}

1. Patel MR, Mahaffey KW, Garg J, et al. Rivaroxaban versus warfarin in nonvalvular atrial fibrillation. N Engl J Med. Sep 8.2011 365:883-891. [PubMed: 21830957]

2. ROCKET AF Study Investigators. Rivaroxaban-once daily, oral, direct factor Xa inhibition compared with vitamin $\mathrm{K}$ antagonism for prevention of stroke and Embolism Trial in Atrial 
Fibrillation: rationale and design of the ROCKET AF study. Am Heart J. Mar.2010 159:340-347. e341. [PubMed: 20211293]

3. Cockcroft DW, Gault MH. Prediction of creatinine clearance from serum creatinine. Nephron. 1976; 16:31-41. [PubMed: 1244564]

4. Wie LJ, Lin DY, Weissfeld L. Regression Analysis of Multivariate Incomplete Failure Time Data by Modeling Marginal Distribution. Journal of the American Statistical Association. 1989:10651073.

5. Guerin A, Lin J, Jhaveri M, Wu EQ, Yu AP, Cloutier M, Gauthier G, Alpert JS. Outcomes in atrial fibrillation patients on combined warfarin \& antiarrhythmic therapy. Int J Cardiol. Jul 31.2013 167:564-569. [PubMed: 22336254]

6. Kerin NZ, Blevins RD, Goldman L, Faitel K, Rubenfire M. The incidence, magnitude, and time course of the amiodarone-warfarin interaction. Arch Intern Med. Aug.1988 148:1779-1781. [PubMed: 3401099]

7. Wan Y, Heneghan C, Perera R, Roberts N, Hollowell J, Glasziou P, Bankhead C, Xu Y. Anticoagulation control and prediction of adverse events in patients with atrial fibrillation: a systematic review. Circ Cardiovasc Qual Outcomes. Nov.2008 1:84-91. [PubMed: 20031794]

8. Steinberg JS, Sadaniantz A, Kron J, et al. Analysis of cause-specific mortality in the Atrial Fibrillation Follow-up Investigation of Rhythm Management (AFFIRM) study. Circulation. Apr 27.2004 109:1973-1980. [PubMed: 15051639]

9. Lafuente-Lafuente C, Longas-Tejero MA, Bergmann JF, Belmin J. Antiarrhythmics for maintaining sinus rhythm after cardioversion of atrial fibrillation. Cochrane Database Syst Rev. 2012; 5:CD005049. [PubMed: 22592700]

10. Daoud EG, Glotzer TV, Wyse DG, Ezekowitz MD, Hilker C, Koehler JL, Ziegler PD. The temporal relationship of atrial tachyarrhythmia, cerebrovascular events, and systemic embolis based on stored device data: a subgroup analysis of TRENDS. Heart Rhythm. 2011 In press.

11. Gnoth MJ, Buetehorn U, Muenster U, Schwarz T, Sandmann S. In vitro and in vivo P-glycoprotein transport characteristics of rivaroxaban. J Pharmacol Exp Ther. Jul.2011 338:372-380. [PubMed: 21515813]

12. Wessler JD, Grip LT, Mendell J, Giugliano RP. The P-glycoprotein transport system and cardiovascular drugs. J Am Coll Cardiol. Jun 25.2013 61:2495-2502. [PubMed: 23563132]

13. Piccini JP, Stevens SR, Lokhnygina Y, et al. Outcomes After Cardioversion and Atrial Fibrillation Ablation in Patients Treated With Rivaroxaban and Warfarin in the ROCKET AF Trial. J Am Coll Cardiol. May 14.2013 61:1998-2006. [PubMed: 23500298]

14. Flaker GC, Hohnloser S, Wojdyla D, Hylek E, Garcia D, Sullivan R, Lopes R, Al-Khatib SM, Granger C. Apixaban Is Efficacious and Safe in Patients with Atrial Fibrillation Using Concomitant Amiodarone: An Analysis from the Aristotle Trial. Journal of the American College of Cardiology. Mar 12.2013 61:E317-E317.

15. Blandino A, Toso E, Scaglione M, Anselmino M, Ferraris F, Sardi D, Battaglia A, Gaita F. Longterm efficacy and safety of two different rhythm control strategies in elderly patients with symptomatic persistent atrial fibrillation. J Cardiovasc Electrophysiol. Jul.2013 24:731-738. [PubMed: 23551460] 


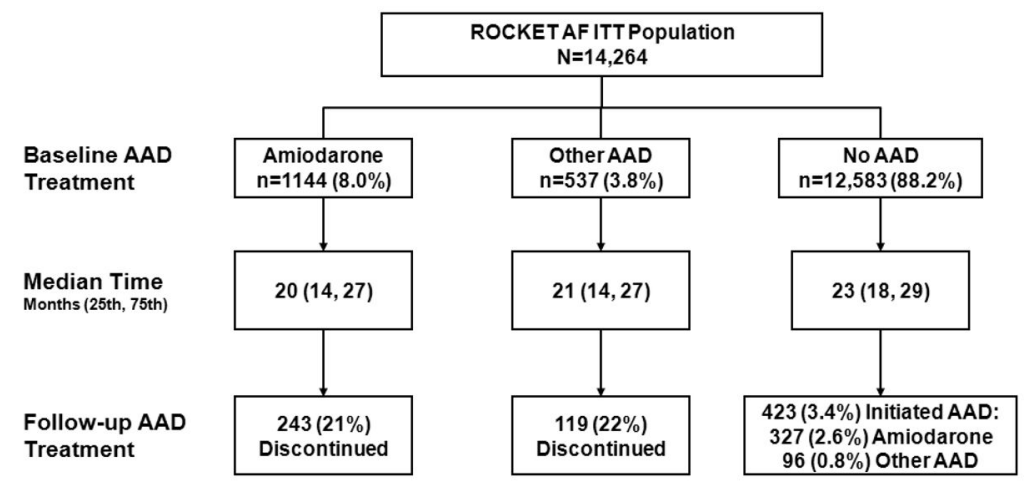

Figure 1.

Derivation of study population and persistence of AAD therapy. Patients were stratified by baseline AAD use: amiodarone, other AAD, or no AAD. AAD=antiarrhythmic drug; ITT=intention-to-treat; ROCKET AF= Rivaroxaban Once Daily Oral Direct Factor Xa Inhibition Compared with Vitamin K Antagonism for Prevention of Stroke and Embolism Trial in Atrial Fibrillation. 


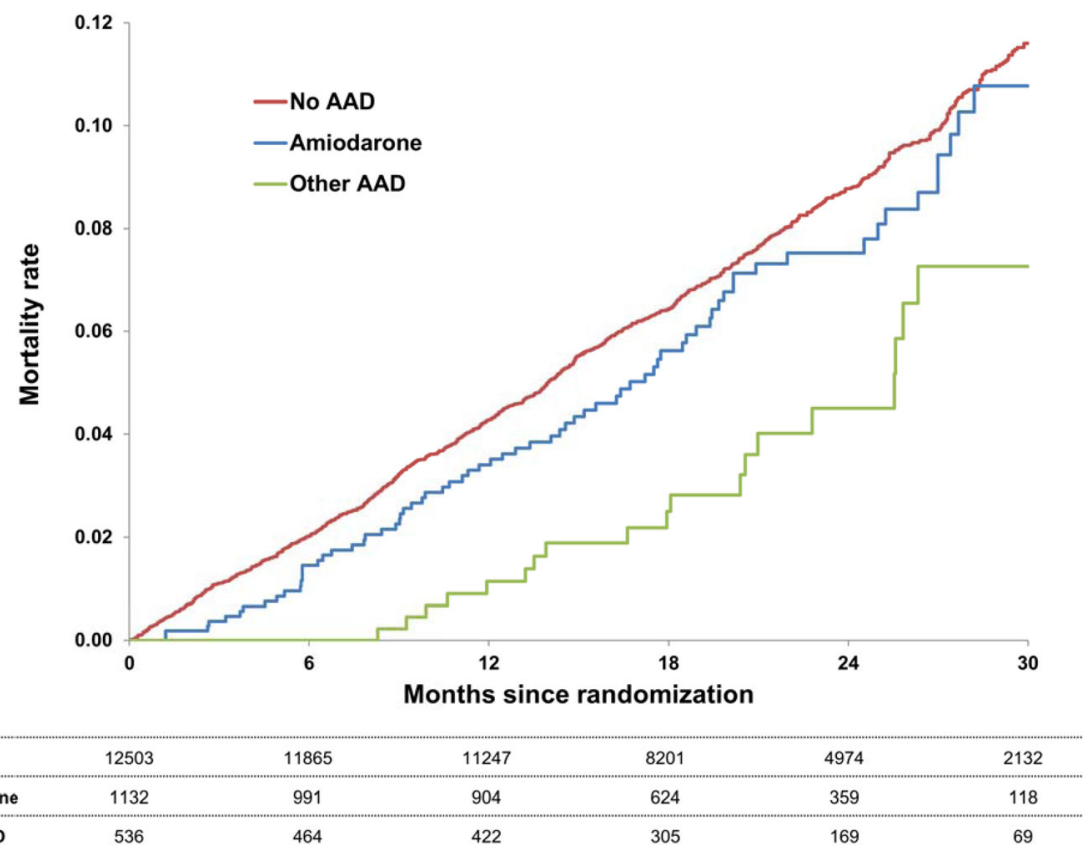

Figure 2.

Kaplan-Meier curves for all-cause mortality, by AAD use at baseline. $\mathrm{P}=\mathrm{NS}$ for all 3 pairwise comparisons, by multivariable Cox models.

$\mathrm{AAD}=$ antiarrhythmic drug. 


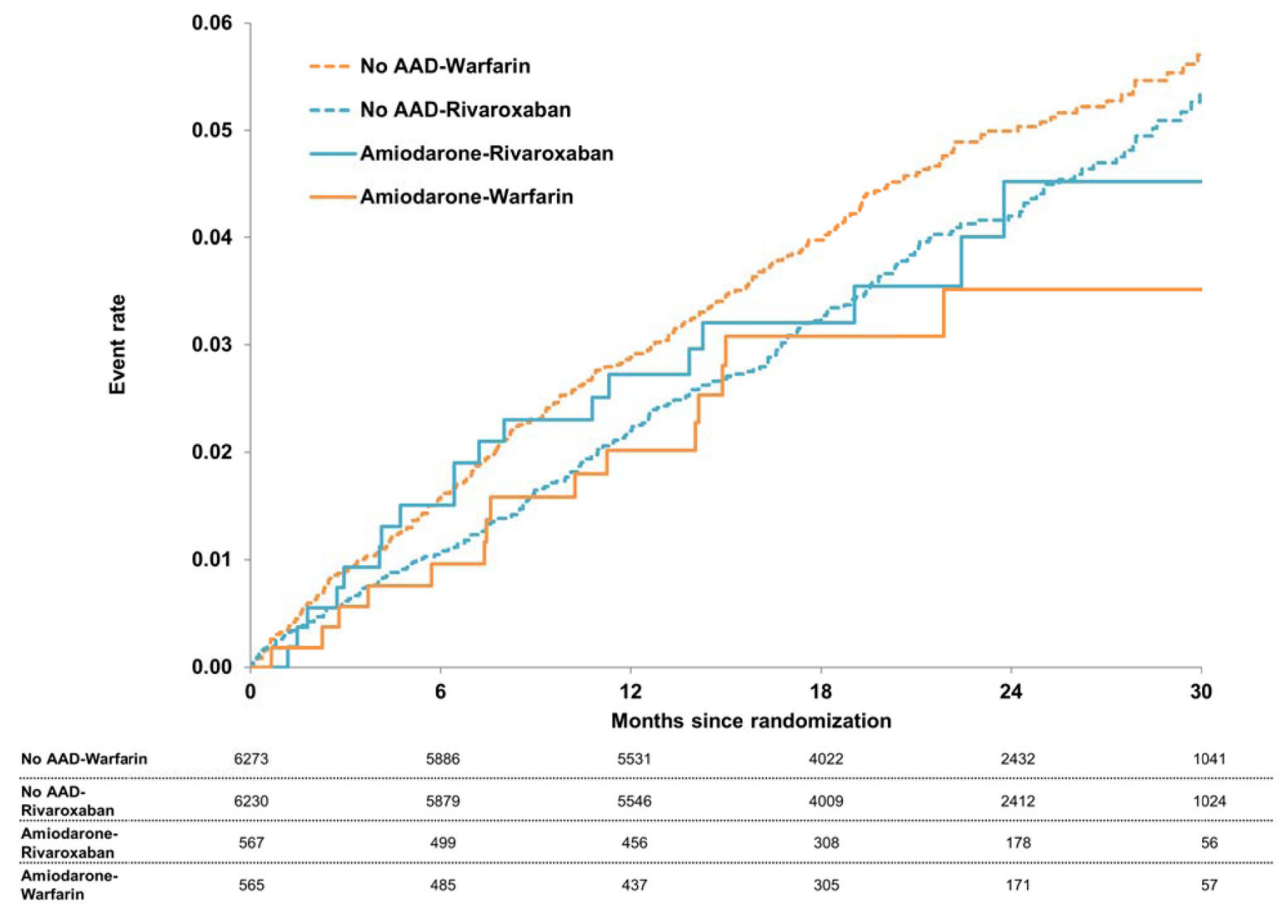

Figure 3.

Kaplan-Meier curves for stroke or non-CNS embolism in patients randomized to rivaroxaban versus warfarin, grouped by amiodarone use at baseline (vs. no AAD). $\mathrm{AAD}=$ antiarrhythmic drug; $\mathrm{CNS}=$ central nervous system. 
Table 1

Baseline characteristics

\begin{tabular}{|c|c|c|c|}
\hline & Amiodarone (N=1144) & Other AAD (N=537) & No AAD $(\mathrm{N}=12,583)$ \\
\hline \multicolumn{4}{|l|}{ Treatment assignment } \\
\hline Rivaroxaban & $50.0 \%(572)$ & $53.1 \%(285)$ & $49.9 \%(6274)$ \\
\hline Warfarin & $50.0 \%(572)$ & $46.9 \%(252)$ & $50.1 \%(6309)$ \\
\hline Age, yrs & $70(61,77)$ & $70(63,76)$ & $73(66,78)$ \\
\hline Female & $38.4 \%(439)$ & $47.5 \%(255)$ & $39.5 \%(4966)$ \\
\hline \multicolumn{4}{|l|}{ Atrial fibrillation } \\
\hline New onset & $1.8 \%(21)$ & $0.2 \%(1)$ & $1.4 \%(180)$ \\
\hline Paroxysmal & $34.4 \%(393)$ & $59.4 \%(319)$ & $14.3 \%(1802)$ \\
\hline Persistent & $63.8 \%(730)$ & $40.4 \%$ (217) & $84.2 \%(10601)$ \\
\hline $\mathrm{CHADS}_{2}$ score, mean (SD) & $3.5(0.9)$ & $3.3(0.9)$ & $3.5(0.9)$ \\
\hline \multicolumn{4}{|l|}{$\mathrm{CHADS}_{2}$ score } \\
\hline 1 & 0 & 0 & $<0.1 \%(3)$ \\
\hline 2 & $10.5 \%(120)$ & $16.6 \%(89)$ & $13.1 \%(1650)$ \\
\hline 3 & $42.7 \%(488)$ & $47.3 \%(254)$ & $43.5 \%(5474)$ \\
\hline 4 & $32.3 \%(370)$ & $25.9 \%(139)$ & $28.5 \%(3582)$ \\
\hline 5 & $12.9 \%(148)$ & $8.8 \%(47)$ & $12.9 \%(1618)$ \\
\hline 6 & $1.6 \%(18)$ & $1.5 \%(8)$ & $2.0 \%(256)$ \\
\hline \multicolumn{4}{|l|}{ Presenting characteristics } \\
\hline $\mathrm{BMI}, \mathrm{kg} / \mathrm{m}^{2}$ & $28.9(25.7,32.7)$ & $28.1(25.0,31.6)$ & $28.1(25.1,31.9)$ \\
\hline Systolic BP, mm Hg & $130(120,140)$ & $130(120,140)$ & $130(120,140)$ \\
\hline Diastolic BP, mm Hg & $80(72,86)$ & $80(70,84)$ & $80(70,85)$ \\
\hline Heart rate, beats/min & $75(65,86)$ & $70(62,80)$ & $76(68,86)$ \\
\hline Creatinine clearance, ${ }^{*} \mathrm{~mL} / \mathrm{min}$ & $67(52,87)$ & $74(57,98)$ & $67(52,86)$ \\
\hline \multicolumn{4}{|l|}{ Baseline comorbidities } \\
\hline Prior ablation for $\mathrm{AF}$ & $2.8 \%(32)$ & $5.8 \%(31)$ & $2.1 \%(258)$ \\
\hline Prior stroke, TIA, or non-CNS embolism & $56.2 \%(643)$ & $67.6 \%(363)$ & $54.1 \%(6805)$ \\
\hline PAD & $5.9 \%(68)$ & $3.4 \%(18)$ & $6.0 \%(753)$ \\
\hline Hypertension & $92.9 \%(1063)$ & $86.2 \%(463)$ & $90.5 \%(11384)$ \\
\hline Diabetes & $39.9 \%(457)$ & $33.9 \%(182)$ & $40.2 \%(5056)$ \\
\hline Prior MI & $16.9 \%(193)$ & $11.0 \%(59)$ & $17.6 \%(2216)$ \\
\hline CHF & $71.1 \%(813)$ & $41.3 \%(222)$ & $62.6 \%(7873)$ \\
\hline COPD & $10.7 \%(122)$ & $8.4 \%(45)$ & $10.6 \%(1330)$ \\
\hline \multicolumn{4}{|l|}{ Medications } \\
\hline Prior VKA use & $52.5 \%(601)$ & $64.4 \%(346)$ & $63.2 \%(7957)$ \\
\hline Prior chronic ASA use & $42.5 \%(486)$ & $32.8 \%(176)$ & $36.1 \%(4543)$ \\
\hline ACE-I/ARB at baseline & $76.9 \%(880)$ & $66.3 \%(356)$ & $74.3 \%(9347)$ \\
\hline Beta blocker at baseline & $50.2 \%(574)$ & $78.6 \%(422)$ & $65.6 \%(8254)$ \\
\hline Digitalis at baseline & $24.0 \%(274)$ & $15.3 \%(82)$ & $40.6 \%(5112)$ \\
\hline Diuretic at baseline & $60.7 \%(694)$ & $41.9 \%(225)$ & $60.2 \%(7571)$ \\
\hline
\end{tabular}

Heart Rhythm. Author manuscript; available in PMC 2015 June 01. 
Continuous variables are shown as median (25th, 75 th percentiles), unless otherwise noted.

* Creatinine clearance calculated according to the Cockcroft-Gault equation.

$\mathrm{AAD}=$ antiarrhythmic drug; $\mathrm{ACE}-\mathrm{I}=$ angiotensin-converting enzyme inhibitor; $\mathrm{ARB}=$ angiotensin $\mathrm{II}$ receptor blocker; $\mathrm{ASA}=$ aspirin; $\mathrm{BMI}=\mathrm{body}$ mass index; $\mathrm{BP}=$ blood pressure; $\mathrm{CHF}=$ congestive heart failure; $\mathrm{CNS}=$ central nervous system; $\mathrm{COPD}=$ chronic obstructive pulmonary disease; $\mathrm{MI}=$ myocardial infarction; $\mathrm{PAD}=$ peripheral arterial disease; $\mathrm{SD}=$ standard deviation; $\mathrm{TIA}=$ transient ischemic attack; $\mathrm{VKA}=\mathrm{vitamin} \mathrm{K}$ antagonist 
Table 2

Anticoagulation control by AAD group among warfarin-treated patients

\begin{tabular}{lccc}
\hline & Amiodarone $(\mathbf{n}=\mathbf{5 5 8})$ & Other AAD $(\mathbf{n = 2 4 6})$ & No AAD $(\mathbf{n}=\mathbf{6 2 2 1})$ \\
\hline TTR, INR 2-3 & $50(33,64)$ & $61(45,74)$ & $58(43,71)$ \\
Time INR $<2$ & $27(16,45)$ & $21(11,37)$ & $24(13,39)$ \\
Time INR 1.5-<2 $(\%)$ & $20(12,29)$ & $15(8,24)$ & $18(11,28)$ \\
Time INR 1-<1.5 $(\%)$ & $4(0,13)$ & $2(0,9)$ & $3(0,9)$ \\
Time INR $<1(\%)$ & $0(0,0)$ & $0(0,0)$ & $0(0,0)$ \\
Time INR $>3$ & $16(9,26)$ & $13(5,21)$ & $13(7,21)$ \\
Time INR $>3-4(\%)$ & $12(6,19)$ & $11(5,17)$ & $11(5,17)$ \\
Time INR $>4-5(\%)$ & $2(0,4)$ & $0(0,2)$ & $1(0,3)$ \\
Time INR $>5(\%)$ & $0(0,1)$ & $0(0,0)$ & $0(0,0)$ \\
\hline
\end{tabular}

Data presented as median percent time (25th, 75th percentiles). P-values for TTR: amiodarone vs. no AAD = <0.0001; other AAD vs. no AAD = 0.16 ; and amiodarone vs. other AAD $<0.0001$ (from pairwise Wilcoxon rank sum tests). A total of $5 \%$ of patients had at least one INR value $<1$; among these patients, the median amount of time spent in this range was $1.1 \%$. A total of $29 \%$ of patients had at least one INR value >5; among these patients, the median amount of time spent in this range was $1.6 \%$.

$\mathrm{AAD}=$ antiarrhythmic drug; INR=international normalized ratio; TTR=time in therapeutic range. 


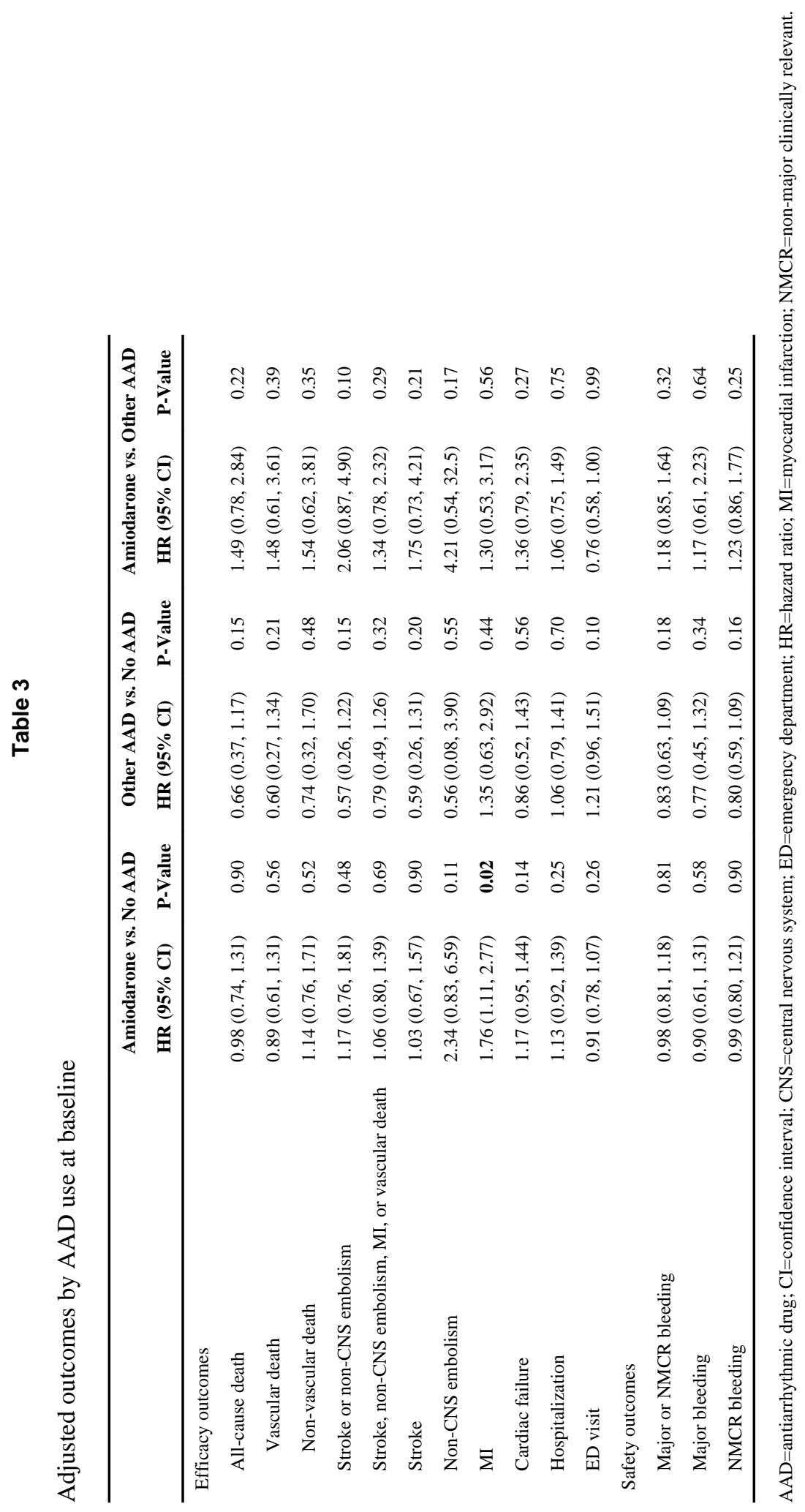

Heart Rhythm. Author manuscript; available in PMC 2015 June 01. 


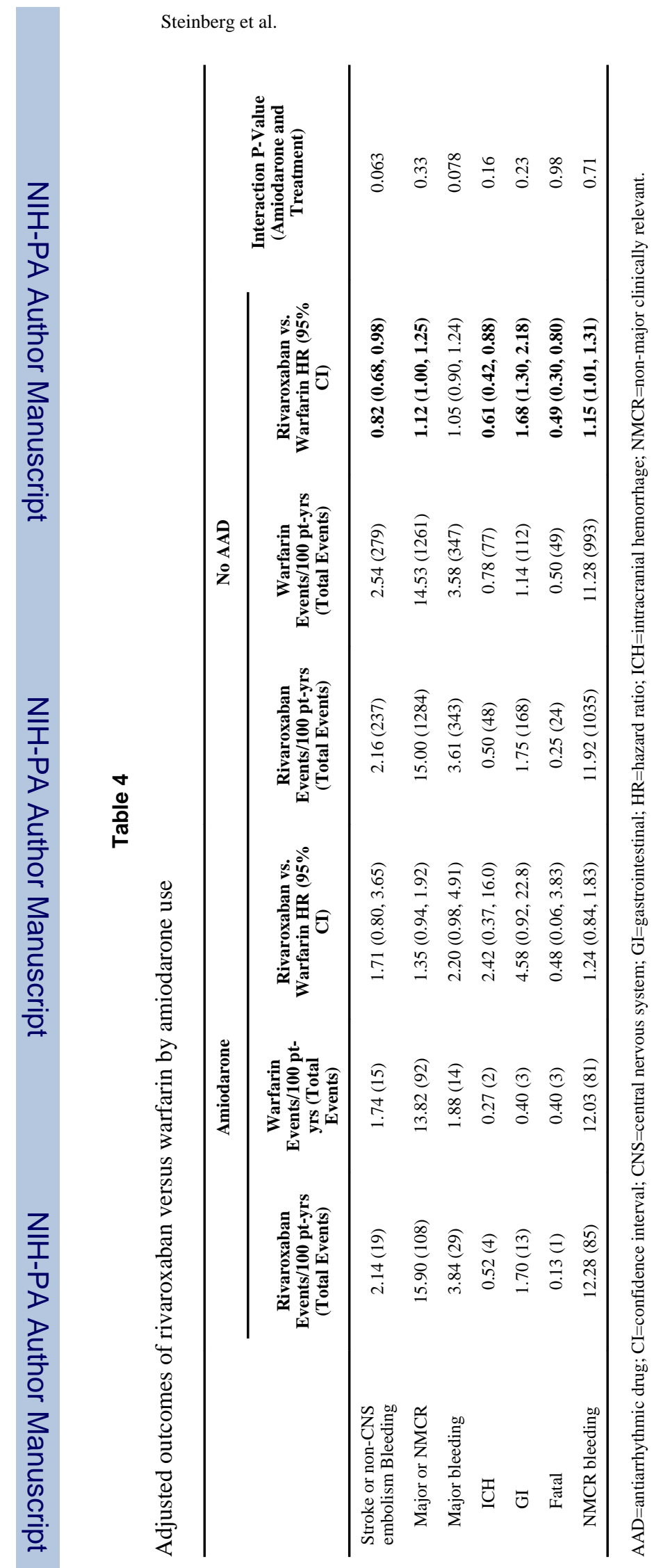

Heart Rhythm. Author manuscript; available in PMC 2015 June 01. 